\title{
First principle study of V-implantation in highly-doped silicon materials
}

\author{
Gregorio García a,b,*, Marcos Casanova-Páez ${ }^{c}$, Pablo Palacios ${ }^{\text {a,d }}$, Eduardo Menéndez-Proupin ${ }^{c}$, \\ Perla Wahnón ${ }^{\mathrm{a}, \mathrm{b}}$ \\ ${ }^{a}$ Instituto de Energía Solar, ETSI Telecomunicación, Universidad Politécnica de Madrid, 28040 Madrid, Spain

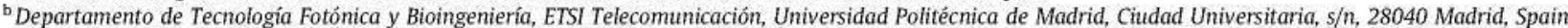 \\ ${ }^{\mathrm{c}}$ Departamento de Física, Facultad de Ciencias, Universidad de Chile, Las Palmeras 3425, 780-0003 Nuñoa, Santiago, Chile \\ ${ }^{\circledR}$ Departamento de Física aplicada a las Ingenierías Aeronáutica y Naval, ETSI Aeronáutica y del Espacio, Pz. Cardenal Cisneros, 3, 28040 Madrid, Spain
}

\begin{abstract}
A B S T R A C T
Density Functional Theory (DFT) has been used to study structural and electronic properties of new compounds based on $\mathrm{V}$-implanted $\mathrm{Si}$ and their potential as infrared photodetectors. Effects derived from the implantation of $\mathrm{V}$ on bulk-Si are calculated at different configurations, i.e., substitutional $\left(\mathrm{V}_{S i}\right)$ and interstitial $\left(V_{i}\right)$ positions as well as the effect of Si vacancies. Despite all implantation processes are energetically penalized, $V_{i}$-implanted compound leads to the lowest formation energies. Furthermore, interstitial implantation in the vicinity of a Si vacancy would lead to a highly favored process. The analysis of the electronic structure shows that $V_{i}$-implanted compounds own an intermediate band (due to $t_{2 g}$ states of vanadium atom), which allows new electronic transitions below $1.0 \mathrm{eV}$. To deal with the bandgap underestimation of common DFT methods, quasiparticle calculations have been applied via the $G_{0} W_{0}$ approximation. Applied correction to the bandgap based on GW has considerably improved theoretical results compared to experimental ones. The investigation of the absorption features points out that the absorption response can be extended up to infrared region via sub-gap transitions across the intermediate band. This work highlights the potential of V-implanted silicon based materials with infrared response.
\end{abstract}

\section{Introduction}

The field of infrared photodetectors has always attracted massive interest due to is potential applications, such as civil security and surveillance, environmental monitoring and telecommunications $[1,2]$. Among others, current infrared photodetection devices are based in mercury cadmium telluride [3] or quantum-dot infrared photodetectors [4]. Even if these materials provide high performances, they suffer some drawbacks, e.g.: high production costs and hardly integration in CMOS (chip complementary metaloxide semiconductor) technology. Silicon based devices assure the low cost and CMOS compatibility criteria. Unfortunately, the band gap of silicon is $1.12 \mathrm{eV}$, which limits its applications at wavelength longer than $1100 \mathrm{~nm}$ [5]. Hence, the modification of silicon band structure is a promising approach to design new Si-based infrared photodetectors.

\footnotetext{
* Corresponding author at: Instituto de Energía Solar and Departamento de Tecnología Fotónica y Bioingeniería, ETSI Telecomunicación, Universidad Politécnica de Madrid, 28040 Madrid, Spain.

E-mail address: ggmoreno@etsit.upm.es (G. García).
}

A potential strategy for extending absorption response of silicon up to infrared region is to incorporate a dopant which creates a partially filled level allowing sub-bandgap absorption. In this sense, different approaches are being explored. Chalcogenhyperdoped silicon based materials have been proven as promising materials for applications in infrared photodetectors due to its absorption features below the Si bandgap [5-8]. Carey et al. [5] and Said et al. [9] investigated the responsivity of photodiodes based on S-hyperdoped silicon pointing out that fabricated photodiodes based on S-hyperdoped Si exhibit magnitude much higher than those measured for non-doped silicon based photodiodes. Similarly, photodiodes based on Se-hyperdoped Si yield improved spectral response up to $0.70 \mathrm{eV}[6,10,11]$. In addition to an extended absorption up to the infrared, a good detectivity of extrinsic photoconductive response under infrared light is also need. Optoelectronic devices based on chalcogen hyperdoped silicon have only demonstrated IR photoresponse at low temperature [12]. Chalcogen hyperdopoing mainly introduces filled (donor) states in the bandgap leading to a high carrier concentration at room-temperature [7,8], which overcomes the sub-bandgap photoconductivity signal at room temperature [12]. Other commonly 
used dopants such as elements of groups III (B, Al and Ga) and V (As and $\mathrm{Sb}$ ) are also limited to low temperatures because of dopant states are thermally ionized at room-temperature [3]. Several approaches have been described to improve the roomtemperature infrared photoresponse. Simmons et al. demonstrated that co-doping the chalcogen donor group (concretely silicon codoped with B and S) can modulate the Fermi level allowing an increase of the infrared photoresponse [13]. The operation temperature may be also increased by doping with deep level impurities which require higher temperatures for thermal ionization [3]. Transition metals introduce deep levels in the bandgap of the host silicon [14]. As matter of fact, Si highly doped with transition elements such as Ti [15-19], V [20], $\mathrm{Ni}[21]$ or $\mathrm{Au}[22,23]$ have demonstrated strong sub-bandgap optical absorption as well as an increase in the high infrared absorption features at room temperature.

All these published works point out that the extended subbandgap response up to infrared regions is related with the ability of studied dopant elements to introduce new level states in the gap of bulk Si. These dopant level states obtained at high concentration (high enough to avoid non-radiative recombination, commonly known as Mott-limit) results in the formation of a continuous energy band inside the bandgap of the host semiconductor known as intermediate band (IB). The so called intermediate band (IB) consists in a partially filled electronic band, placed into the host semiconductor gap and isolated from the valence band (VB) and the conduction band $(\mathrm{CB})$. Intermediate band has to have a small dispersion and must not be a discrete level, whereas it has to be narrow enough to be well isolated from both $\mathrm{VB}$ and $\mathrm{CB}$. Thereby, in addition to prompting electrons from $\mathrm{VB}$ to $\mathrm{CB}$ (VB-CB), transitions from the VB to IB (VB-IB) and from IB to CB (IB-CB) by absorbing photons with lower energy than the bandgap are also allowed. As a result, IB materials are attracting increasing interest towards developing new photovoltaic materials with an improved efficiency $[19,24,25]$. These same features also make intermediate band materials suitable for their use in infrared photodetectors [5-11,22].

Designing new electronic devices using doped-Si with the desired features present several challenges, such as crystallinity, optical absorbance and dopant concentration as well as to explore adequate doping elements that could modulate absorption features. Previous works shown that materials based on doped-Si with transition metals such as $\mathrm{Ti}$ [15-19], Ni [21], Au [22,23] or Co [26] would lead to the formation of a new band within the gap of Si host semiconductor. Recently, an extended photoresponse up to the infrared region for vanadium hyperdoped silicon obtained by ion implantation has been reported [20]. Our work explores the effect of introducing vanadium to explain the formation of an intermediate band with the desired properties by using quantum calculations. For this purpose, structural, energetic and electronic properties of different $\mathrm{V}$-implanted $\mathrm{Si}$ are studied in detail through quantum calculations. On the other hand, defects comprising vacancies and interstices are common in natural materials. Such defects can be innate or created during the material manufacturing process. In semiconductors the presence of (undesired) defects can modify their electrical and optical properties $[27,28]$. Hence, models of $\mathrm{V}$-implanted silicon compounds with $\mathrm{Si}$ vacancies were also studied. To our knowledge, studies on the simultaneous effect of $\mathrm{V}$-implantation and defects from a theoretical point of view are missing in literature.

Most of the calculations available in the literature for this kind of materials have been based on DFT in the Kohn-Sham implementation with LDA (Local Density Approximation) or GGA (Generalized Gradient approximation) schemes for the exchangecorrelation functional, due to their efficiency to deduce structural, electronic and other properties of a multitude of condensed matter systems. Unfortunately, it is well known that these functionals fail to correctly predict the bandgap [29], which is an hindrance to research in fields including semiconductors, optical or photovoltaic materials. To overcome the "bandgap problem", we employ manybody perturbation theory in $G W$ approximation to calculate quasiparticle self-energy corrections for the electronic states, which yields results that are in very good agreement with experiments for a wide range of materials [30].

\section{Theoretical details}

Density Functional Theory (DFT) simulations were carried out using the generalized gradient approximation of Perdew, Burke and Ernzerhof (PBE) [31] as implemented in the VASP code $[32,33]$, along to projector augmented wave (PAW) potentials to represent the inert core electrons $[34,35]$. Structural relaxations were done by conjugate gradients, with a convergence criteria that forces acting on all atoms do not exceed $0.01 \mathrm{eV}^{-1}$. Structural optimization were done using a $k$-point mesh of $6 \times 6 \times 6$ in the Monkhorst-Pack scheme [36]. A Methfessel-Paxton first-order scheme with $0.1 \mathrm{eV}$ of smearing was used to extract the occupations of Kohn-Sham eigenvalues [37]. The tetrahedron method was applied to obtain electronic density of states (DOS) [38]. Model bulk-Si was described as supercell $2 \times 2 \times 2$ of $\mathrm{Si}_{8}$ cubic unit cell (i.e., 64 atoms, labeled as $\mathrm{Si}_{64}$ ). The structures derived from the former due to the presence of $\mathrm{V}$ atoms and/or $\mathrm{Si}$ vacancies were relaxed in order to study the effects of $\mathrm{V}$ implantation and/or Si vacancies on structural and electronic properties. In this way, one $\mathrm{V}$ atom leads to a concentration $[\mathrm{V}] \gg 10^{20} \mathrm{~cm}^{-3}$, which considerably surpasses the Mott-limit. Nevertheless, it is expected that the electronic structure for such dilution would elucidate whether an isolated IB can be obtained or if the energy levels from $3 d$ electrons overlap with VB and/or CB of bulk-Si, except that the bandwidth of the IB would be slightly increased [19,39].

Optical properties were assessed by means of the absorption coefficient derived from the dielectric functions as implemented in VASP code. Thus, the imaginary part was obtained as the sum of independent transitions between Kohn-Sham states, without local field effects, while the real part was obtained from the imaginary part by the Kramers-Krönig relations. To get converged frequency-dependent dielectric properties, approximately 250 bands and an $8 \times 8 \times 8$ sampling of the Brillouin zone were needed. Since the imaginary part of the dielectric function has been analyzed as a simple sum over independent transitions, we can separate it into the contributions from different transitions. This analysis was carried out by using a home-modified version of the original OPTICS code developed by Furthmüller [40].

As known, standard-DFT methods underestimate the bandgap of the semiconductor due to an inaccurate treatment of the excited states. Hence, a correction has been applied to correct the GGA underestimation of the band gap. Different approximations have been applied to solving the DFT bandgap, such as the application of a scissor operator over the empty states to fit the experimental bandgap of the host semiconductor, time-dependent DFT, or hybrid and screened hybrid functional or quasi particle energy calculations $[19,29,39]$. Concretely, many-body perturbation theory within the $G W$ approach was applied. The GW approach is based on a dynamic dielectric screening of the Coulomb potential, while the electronic self energy is approximated by a convolution in terms of the Green's function $G$ and the screened interaction $W$. In this paper, GW calculations were carried out to correct DFT eigenvalues without further interactions, i.e., $G_{0} W_{0}$ approach [30], wherein the calculations start from DFT eigenvalues and eigenfunctions to obtain many-body GW self energy. This method yields successfully agreement with experiment results for intermediate 
band materials, within the limits of the approach $[41,42]$. Unfortunately, GW approximation is very time consuming. Thus, quasi particle energies were only computed for $\mathrm{Si}_{64}$ and $\mathrm{V}_{i}$-implanted compound without vacancies just at the $\Gamma$-point through the $G_{0} W_{0}$ approach. Our results from $G_{0} W_{0}$ approximation at the $\Gamma$ point yields improved bandgap energies for bulk-Si (see Section 3.4).

\section{Results}

\subsection{Main structural parameters}

This work includes the study for bulk-Si (based on $\mathrm{Si}_{64}$ model) and $\mathrm{V}$-implanted compounds in substitutional $\left(\mathrm{V}_{S_{i}}\right)$ and interstitial $\left(V_{i}\right)$ cases. Fig. 1 sketches the applied procedure to built unit cells for V-implanted compounds here studied, while the most important parameters of $\mathrm{Si}_{64}$ and $\mathrm{V}$-implanted compounds are gathered in Table 1 . Bulk-Si was described as a supercell $2 \times 2 \times 2$ of $\mathrm{Si}_{8}$ cubic unit cell with 64 atoms labeled as $\mathrm{Si}_{64}$. The optimized structure for Bulk-Si (described as a supercell $2 \times 2 \times 2$ of $\mathrm{Si}_{8}$ cubic unit cell with 64 atoms, $\mathrm{Si}_{64}$ ) yields a lattice parameter $a=5.465 \AA$, while the interatomic distances between $\mathrm{Si}$ atoms are $2.366 \hat{\AA}$. The departure of the lattice cell $a$ and $\mathrm{Si}-\mathrm{Si}$ bond length (d[Si$\mathrm{Si}])$ from the experimental values $(a=5.431 \AA$, d $[\mathrm{Si}-\mathrm{Si}]=2.352 \AA$ ) [43] turn out to be $\approx 0.6 \%$. This slight overestimation of the lattice cell agrees with the expected behavior of GGA functionals.

From the optimized structure for $\mathrm{Si}_{64}, \mathrm{~V}$-implanted compound in substitutional position was built by placing one vanadium at the position of one $\mathrm{Si}\left(\mathrm{ii} . \mathrm{V}_{\mathrm{Si}_{i}} \mathrm{Si}_{63}\right)$. The lattice parameter is increased up to $a=5.471 \AA$. This substitution also causes an increment of $0.065 \AA$ in the distance to the four nearest $\mathrm{Si}$ atoms (d[V-Si] $=2.431 \AA$ ), while the distance for the farthest pair of $\mathrm{Si}$ atoms from $\mathrm{V}_{S i}$ is $2.368 \AA$. The four nearest $\mathrm{Si}$ atoms are tetrahedrally coordinated to the $\mathrm{V}$ atom. The increment of both lattice cell parameter and d[V-Si] with regard to d[Si-Si] for $\mathrm{Si}_{64}$ could be related to the fact that $\mathrm{V}$ atom owns a larger atomic radii than $\mathrm{Si}$ one $(1.740 \AA$ and $1.110 \AA$ for $\mathrm{V}$ and $\mathrm{Si}$, respectively). Hence, $\mathrm{V}_{S i}$-implantation does not considerable modify the bulk-Si structure. Similarly, $V_{i^{-}}$ implanted compound at interstitial position in absence of Si vacancies (iii. $\mathrm{V}_{i} \mathrm{Si}_{64}$ ) was also generated from $\mathrm{Si}_{64}$ by placing a $\mathrm{V}$ atom at the center of a tetrahedron formed by four $\mathrm{Si}$ atoms. The relaxed unit cell for $\mathrm{V}_{i}$-Si compound in absence of $\mathrm{Si}$ vacancies leads to a disposition wherein $\mathrm{V}$ atom is tetrahedrally coordinated to the four nearest neighbors $\mathrm{Si}$ atoms with a distance $\approx 2.440 \AA$, while the six second nearest atoms are placed of around $2.746 \AA$ in an octahedrical configuration (see Fig. 1S). Note that the void of the tetrahedron defined by four $\mathrm{Si}$ atoms as well as the void of the octahedral defined by the six second nearest $\mathrm{Si}$ atoms are enough to allocate $\mathrm{V}$ atom without steric hindrance with $\mathrm{Si}$ atoms. Although, at short range (i.e. in the vicinity of vanadium atom) Si-Si bond-lengths are

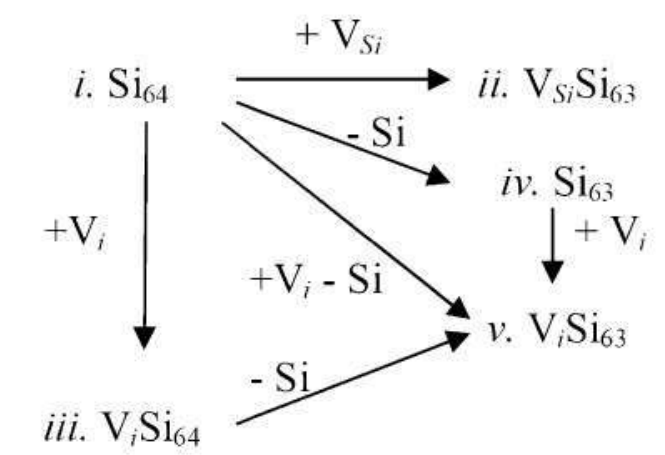

Fig. 1. Applied protocol to build different structures studied in this work. slightly modified respect to bulk $\mathrm{Si}$ (studied as $\mathrm{Si}_{64}$ ), this effect decreases with the distance respect to vanadium atom. Hence, at long range $\mathrm{d}[\mathrm{Si}-\mathrm{Si}]$ and lattice parameter tend to be similar than those obtained for bulk $\mathrm{Si}$. As seen below, the crystalline field is mainly due to the octahedrical environment. As concerns as the effect of $\mathrm{V}_{i}$-implantation on the main unit cell parameters of the host $\mathrm{Si}_{64}$, both lattice parameter $a$ and d[Si-Si] (measured between two $\mathrm{Si}$ atoms located in the available farthest positions) keep constant or vaguely affected.

Before investigating $\mathrm{V}_{i}$-implanted compounds in presence of $\mathrm{Si}$ vacancies $\left(v . \mathrm{V}_{i} \mathrm{Si}_{63}\right)$, main structural parameters of the system labeled as $i v$. $\mathrm{Si}_{63}$ (i.e., bulk-Si with vacancies) are briefly assessed. Such information could be useful to rationalize some structural effects noted for $\mathrm{V}_{i} \mathrm{Si}_{63}$ compounds. $\mathrm{Si}_{63}$ compound was obtained by removing one $\mathrm{Si}$ atom from relaxed $\mathrm{Si}_{64}$. From its unit cell parameters reported in Table 1 is inferred that lattice parameter is reduced with regard to bulk-Si ( $a$ is reduced from $5.465 \AA$ up to $5.443 \AA$ ), while $d[\mathrm{Si}-\mathrm{Si}]$ at the remotest position of the vacancy also becomes somewhat shorter (there is a shortening equal to $0.006 \AA$ ). This is indicative that $\mathrm{Si}$ vacancies have larger effects on the bulk-Si structure than $\mathrm{V}$ implantation either interstitial or substitutional position.

Finally, $V_{i}$-implanted compounds with one $\mathrm{Si}$ vacancy $\left(\mathrm{V}_{i} \mathrm{Si}_{63}\right)$ were studied. For this purpose, several models with different relative dispositions between $\mathrm{V}$ atom and $\mathrm{Si}$ vacancy were optimized. Thus, three different relative positions between $V_{i}$ atom and $\mathrm{Si}$ vacancy were defined: the closest position between $V_{i}$ atom and Si vacancy, the farthest position between them as well as an intermediate position (labeled as a), c) and b), respectively). Fig. 2S shows optimized structure of $\mathrm{V}_{i} \mathrm{Si}_{64}$ compound, which is here used to define each configuration. Configuration a) is obtained by removing the $\mathrm{Si}$ atom nearest to $\mathrm{V}$, i.e., one silicon atom tetrahedrically coordinated (even though similar results are obtained for a $\mathrm{Si}$ atom octahedrically coordinated). In this case, the initial distance between $\mathrm{Si}$ vacancy and $\mathrm{V}$ atom is ranged between $2.440 \AA$ and $2.476 \AA$. Based on a $2 \times 2 \times 2$ supercell, the largest available distance between $\mathrm{V}$ and one $\mathrm{Si}$ atom is $8.22 \mathrm{~A}$, which was used to define our model with the farthest disposition between $\mathrm{V}$ and $\mathrm{Si}$ vacancy (model c)). Note that the average distance between the farthest and the closest positions above defined is $\approx 5.33 \AA$. In this sense, there are some $\mathrm{Si}$ atoms whose $\mathrm{Si}-\mathrm{V}$ lengths are $\approx 4.76 \AA$. Thus, removing one of these atoms was used to define $\mathrm{V}_{i} \mathrm{Si}_{63}$ with an intermediate distance between $\mathrm{V}$ atom and $\mathrm{Si}$ vacancy (labeled as b)). In addition, as shown in Fig. $1, V_{i}$-implanted compounds with one $\mathrm{Si}$ vacancy were obtained $i$ ) from bulk $\mathrm{Si}\left(\mathrm{Si}_{64}\right)$ by the simultaneous addition and removal of one $\mathrm{V}$ and $\mathrm{Si}$ atom, respectively; ii) from optimized structure of $\mathrm{V}_{i} \mathrm{Si}_{64}$ removing one $\mathrm{Si}$ atom; iii) from optimized structure of bulk $\mathrm{Si}$ with defects $\left(\mathrm{Si}_{63}\right)$ by adding one $V_{i}$ atom. For the sake of clarity, our analysis will be only focused on the structures of minimal energy obtained for each model, although similar results are obtained instead of the selected initial structure. This procedure warrants that results here reported are referred to the most stable configurations of $\mathrm{V}_{i} \mathrm{Si}_{63}$.

For $\mathrm{V}_{i}$-implanted compounds in presence of one $\mathrm{Si}$ vacancy at the shortest distance between them, we firstly analyze the tendency of $\mathrm{V}$ atom to occupy the vacancy place (leading to $\mathrm{V}_{5 i} \mathrm{Si}_{63}$ ). Our results point out that $\mathrm{V}$ does not displace up to the vacancy since optimized structures similar to $\mathrm{V}_{S i} \mathrm{Si}_{63}$ were not obtained when $v-a) \mathrm{V}_{i} \mathrm{Si}_{63}$ models are used as starting point. But after the optimization, the closest $\mathrm{Si}$ atom (which would match with $\mathrm{Si}$ atom defined as the intermediate position in Fig. 2S) moves up to fill the vacancy near to $\mathrm{V}$ atom. Therefore, after relaxing initial structure of $v-a) \mathrm{V}_{i} \mathrm{Si}_{63}$ the $\mathrm{Si}$ vacancy is located in an analogous position than those obtained for $v-b) V_{i} S_{63}$ relaxed structure, which agrees with the similarity of results here reported for both $v-a) \mathrm{V}_{i} \mathrm{Si}_{63}$ and $v-b$ ) $\mathrm{V}_{i} \mathrm{Si}_{63}$ compounds. Both $\left.v-a\right) \mathrm{V}_{i} \mathrm{Si}_{63}$ and $\left.v-b\right) \mathrm{V}_{i} \mathrm{Si}_{63}$ yield a lattice 
Table 1

Main structural parameters obtained from the relaxation carried out for selected compounds and the corresponding formation energies ( $E$ ).

\begin{tabular}{|c|c|c|c|c|}
\hline Compound & $a(\AA)$ & $\mathrm{d}[\mathrm{V}-\mathrm{Si}]^{\mathrm{a}}(\AA)$ & $\mathrm{d}[\mathrm{Si}-\mathrm{Si}]^{\mathrm{b}}(\mathrm{A})$ & $E^{f}(\mathrm{eV})$ \\
\hline i. $\mathrm{Si}_{64}$ & 5.465 & & 2.366 & \\
\hline ii. $\mathrm{V}_{5 \mathrm{~S}} \mathrm{Si}_{63}$ & 5.471 & 2.431 & 2.368 & 3.06 \\
\hline iii. $\mathrm{V}_{1} \mathrm{Si}_{64}$ & 5.465 & $2.440 / 2.746$ & 2.367 & $0.97^{\mathrm{d}}$ \\
\hline iv. $\mathrm{Si}_{63}$ & 5.443 & & 2.360 & $3.55^{e}$ \\
\hline$v-a . V_{i} S_{63}$ & 5.435 & $2.408 / 2.439 / 2.736 / 2.602$ & 2.362 & $2.89^{f}$ \\
\hline v-b. $V_{i} S_{63}$ & 5.435 & $2.408 / 2.439 / 2.738 / 2.601 /$ & 2.362 & $2.89^{\prime}$ \\
\hline$v-c . V_{i} S_{i} i_{63}$ & 5.437 & $2.483 / 2.769$ & 2.356 & $2.25^{\mathrm{f}}$ \\
\hline
\end{tabular}

a The shortest distance between $\mathrm{V}$ and $\mathrm{Si}$ atoms.

${ }^{b}$ Distance between $\mathrm{Si}$ atoms located as far as possible of $\mathrm{V}$ atom.

c Estimated as defined in Eq. (1).

d Estimated as defined in Eq. (2).

e Estimated as defined in Eq. (3).

${ }^{\mathrm{f}}$ Estimated as defined in Eq. (4).

parameter $a=5.435 \AA$, while the distance between $\mathrm{V}$ atom and the four first nearest $\mathrm{Si}$ atoms lies in the range $2.439 \AA$ and $2.408 \AA$. This distance is ranged between $2.601 \AA$ and $2.738 \AA$ for the second nearest $\mathrm{Si}$ atoms. When $\mathrm{V}$ atom is implanted at the farthest position, lattice cell parameter $a=5.437 \AA$, while the first four closest $\mathrm{Si}$ atoms and the second nearest $\mathrm{Si}$ atoms are located at $2.483 \AA$ and $2.769 \AA$ from $V$ atom. Instead of the relative disposition between $\mathrm{V}$ atom and $\mathrm{Si}$ vacancy, optimized structures of $\mathrm{V}_{i} \mathrm{Si}_{63}$ lead to the shortest distances between $\mathrm{Si}$ atoms $(\mathrm{d}[\mathrm{Si}-\mathrm{Si}]=2.360 \AA)$. Note that similar effects are noted for $\mathrm{Si}_{63}$; hence this distortion would be mainly due to the Si vacancy. Based on unit cell parameter $a$, it can be also concluded that the presence of both $V$ atom and Si vacancy leads to the largest cell distortion, even at long range. Albeit two sets of $\mathrm{Si}$ atoms are also found around $\mathrm{V}$ as a function of the $\mathrm{V}$-Si distance for $\mathrm{V}_{i}$-implanted $\mathrm{Si}$ with one $\mathrm{Si}$ vacancy, the presence of a Si vacancy distorts the tetrahedrical and octahedrical coordination of $\mathrm{V}$ atom above described for $\mathrm{V}_{i^{-}}$ implanted Si without vacancies. As expected, this distortion decreases with the distance between $\mathrm{V}$ and $\mathrm{Si}$ vacancy. As seen below, the presence of one vacancy has not only important effects on the structural parameters.

\subsection{Formation energies}

Aimed at obtaining some information about the stability of different V-implanted Si compounds here studied, formation energies $\left(E^{f}\right)$ for optimized compounds were estimated from the total energies. Several papers have been published dealing with the calculation of formation energies in doped systems by using DFT calculations. Such papers are devoted to investigate properties for a single, isolated point defect or impurity in a semiconductor $[28,44]$. However, it is well known that the concentration of impurities should exceed the so-called Mott-Limit to form an intermediate band $[15,45]$. As matter of fact, several papers have shown that a supersaturated Si based material with very high dopant concentration above the equilibrium solubility limit is needed to obtain an IB $[16,21-23,46]$. In this work formation energies were estimated in concordance with previous works dealing with implanted Si-compounds $[19,39]$ and other IB materials previously studied by our group [47-50]. Thus, formation energies $\left(E^{f}\right)$ were defined as:

$$
\begin{aligned}
& E^{f}\left[V_{S i} S i_{63}\right]=E\left[V_{S i} S i_{63}\right]-\left(\frac{63}{64} E\left[S i_{64}\right]+\frac{1}{2} E\left[V_{2}\right]\right)=3.06 \mathrm{eV} \\
& E^{f}\left[V_{i} S i_{64}\right]=E\left[V_{i} S i_{64}\right]-\left(E\left[S i_{64}\right]+\frac{1}{2} E\left[V_{2}\right]\right)=0.97 \mathrm{eV} \\
& E^{f}\left[S i_{63}\right]=\left(E\left[S i_{63}\right]+\frac{1}{64} E\left[S i_{64}\right]\right)-\left(E\left[S i_{64}\right]\right)=3.55 \mathrm{eV}
\end{aligned}
$$

$$
\begin{aligned}
E^{f}\left[V_{i} S i_{63}\right] & =E\left[V_{i} S i_{63}\right]-\left(\frac{63}{64} E\left[S i_{64}\right]+\frac{1}{2} E\left[V_{2}\right]\right) \\
& =2.89 \mathrm{eV}, 2.89 \mathrm{eV}, 2.25 \mathrm{eV}
\end{aligned}
$$

where $E\left[S i_{64}\right], E\left[V_{S S} S i_{63}\right], E\left[V_{i} S i_{64}\right], E\left[S i_{63}\right], E\left[V_{i} S i_{63}\right]$ and $E\left[V_{2}\right]$ stand for the total energies of $\mathrm{Si}_{64}, \mathrm{~V}_{S i}$-implanted $\mathrm{Si}\left(\right.$ ii. $\left.\mathrm{V}_{S i} \mathrm{Si}_{63}\right), \mathrm{V}_{i}$-implanted Si without vacancies (iii. $\mathrm{V}_{i} \mathrm{Si}_{64}$ ), $\mathrm{Si}_{63}, \mathrm{~V}_{i}$-implanted $\mathrm{Si}$ with one vacancy $\left(v . \mathrm{V}_{i} \mathrm{Si}_{63}\right)$ and $\mathrm{BCC}$ crystalline structure of Vanadium (made up of two atoms per formula). The formation energy for substitutional implantation yields the highest value $\left(E^{f}\left[\mathrm{~V}_{5 i} \mathrm{Si}_{63}\right]=3.06 \mathrm{eV}\right)$, while interstitial implantation leads to $E^{f}\left[\mathrm{~V}_{i} \mathrm{Si}_{64}\right]=0.97 \mathrm{eV}$. Therefore, although both implantation types bring out high energy penalization per vanadium inserted, this energy change is considerable lower $(2.09 \mathrm{eV})$ for $\mathrm{V}_{i}$-implanted compound. In the case of $\mathrm{V}_{i^{-}}$ implanted compounds with one silicon vacancy, formation energy becomes $E^{f}\left[\mathrm{~V}_{i} \mathrm{Si}_{63}\right]=2.89 \mathrm{eV} / 2.89 \mathrm{eV} / 2.25 \mathrm{eV}$ (for the shortest $\mathrm{V}$ vacancy/intermediate $\mathrm{V}$-vacancy/the largest $\mathrm{V}$-vacancy distance). Even in presence of one Si vacancy, interstitial implantation of $\mathrm{V}$ atom is more favorable than substitutional positioning. As concerns as the effect of the relative disposition between $\mathrm{V}_{i}$ atom and $\mathrm{Si}$ vacancy, the structure with the farthest distance between $\mathrm{V}$ atom and $\mathrm{Si}$ vacancy yields the smallest formation energy. Note that the formation energy of one silicon vacancy also implies a high formation energy value $\left(E\left[\mathrm{Si}_{63}\right]=3.55 \mathrm{eV}\right)$, which is the same order than the formation energies of $\mathrm{V}$-implanted compounds in presence of $\mathrm{Si}$ vacancies. Thus, formation energies corresponding to $\mathrm{V}_{S i} \mathrm{Si}_{63}$ and $\mathrm{V}_{i^{-}}$ $\mathrm{Si}_{63}$ compounds would be mainly due to the formation energy of one silicon vacancy. Hence, formation energies of $\mathrm{V}_{S i}$ implanted compound (ii. $\left.\mathrm{V}_{S i} \mathrm{Si}_{63}\right)$ and $\mathrm{V}_{i}$-implanted silicon $\left(\nu . \mathrm{V}_{i} \mathrm{Si}_{63}\right)$ with one vacancy can be also calculated considering the presence of one $\mathrm{Si}$ vacancy before $\mathrm{V}$ implantation:

$$
\begin{aligned}
E^{f}\left[V_{S i} S i_{63}\right] & =E\left[V_{S i} S i_{63}\right]-\left(E\left[S i_{63}\right]+\frac{1}{2} E\left[V_{2}\right]\right)=-0.49 \mathrm{eV} \\
E^{f}\left[V_{i} S i_{63}\right] & =E\left[V_{i} S i_{63}\right]-\left(E\left[S i_{63}\right]+\frac{1}{2} E\left[V_{2}\right]\right) \\
& =-0.66 \mathrm{eV},-0.66 \mathrm{eV},-1.30 \mathrm{eV}
\end{aligned}
$$

As expected, $\mathrm{V}$ implantation at interstitial positions would be again the most favorable process. Note that $V_{i}$ implantation in the remotest (available) position respect to one Si vacancy entails formation energies much lower than zero, i.e., such process would be energetically more favored. Formation energies defined according Eqs. (5) and (6) can provide useful information about the tendency of $V$ atom to be placed in the vicinity of one Si vacancy. As said, our analysis on the tendency of $\mathrm{V}$ atom to move to the $\mathrm{Si}$ vacancy place in $v-a . V_{i} \mathrm{Si}_{63}$ only revealed that for this situation the vacancy is preferably filled by the closest $\mathrm{Si}$ atom. This points 
out that the migration of one Si atom to the vacancy is more favorable, which also allows larger remoteness between Si vacancy and interstitial vanadium. This fact is also in agreement with the lowest formation energy calculated for $v-c . V_{i} \mathrm{Si}_{63}$.

We are aware that vacancy concentrations here studied could be higher than those ones present in experimental samples. Consequently, formation energy defined by Eq. (2) could be extrapolated to those situations wherein the vacancy (in a low concentration) is so far from the $\mathrm{V}$ that its topology could be similar than those described for iii. $\mathrm{V}_{i} \mathrm{Si}_{64}$. However, accordingly with formation energy defined by Eq. (6), V atom would tend to allocate relatively close to one Si vacancy with a topology similar than those reported for $v-c . V_{i} S_{63}$. Hence, real sample of $V$-implanted silicon compound should be qualitatively described as a combination of structures optimized for iii. $\mathrm{V}_{i} \mathrm{Si}_{64}$ and $v-c . \mathrm{V}_{\mathrm{i}} \mathrm{Si}_{63}$, with a small contribution from substitutional implantation (ii. $\mathrm{V}_{S \mathrm{~S}} \mathrm{Si}_{63}$ ).

\subsection{Electronic structure}

For simplicity, discussions on the bulk-Si electronic structure were not deeply done herein as they have been extensively studied in the literature $[19,51,52]$. In short, our results are in agreement with the available data, which yields a theoretical band gap equal to $0.63 \mathrm{eV}$. As expected, this value is underestimated compared with the experimental one $(1.12 \mathrm{eV})$, which makes necessary to correct it, in order to obtain appropriate optical spectra. As discussed below, upon the applying of a rigid shift based on $G_{0} W_{0}$ calculations (see Section 2), more accurate theoretical bandgap are obtained (1.03 eV). Figs, 2, 3 and 3S plots density of states (projected density of states of the transition metal are also displayed) and band structure of studied V-implanted compounds. The presence of vanadium leads to $\mathrm{V}$-implanted compounds with a spin polarized electronic structure. Based on formation energies above calculated, interstitial implantation should be the most favored process. Hence, results here exposed are mainly focused on $V_{i^{-}}$ implanted compounds. In brief, for $\mathrm{V}_{S i^{-}}$-implanted compound $\left(\mathrm{V}_{S i^{-}}\right.$ $\mathrm{Si}_{63}$ ), the bandgap for bulk-Si is considerably opened up to $1.07 \mathrm{eV}(1.37 \mathrm{eV}) / 1.61 \mathrm{eV}(1.91 \mathrm{eV})$ for spin up/down channel due to vanadium bands (at $\Gamma$-point). Furthermore, important transitions with energies ranged between $0.70 \mathrm{eV}(1.00 \mathrm{eV})$ and $1.29 \mathrm{eV}(1.59 \mathrm{eV})$ should be also mentioned. See supplementary information for a more detailed description.

For interstitial configuration in absence of $\mathrm{Si}$ vacancies $\left(\mathrm{V}_{i} \mathrm{Si}_{64}\right.$, Fig. 2), the effect of the octahedral crystal field originated by the six second nearest $\mathrm{Si}$ atoms splitting $3 d$ levels into a low energy $t_{2 g}$ triplet and a high-energy $e_{g}$ doublet. The most obvious change is the appearance of an additional intermediate band (IB) within the bandgap of the host semiconductor (due to the low energy $t_{2 g}$ triplet for spin down) with a bandwidth equal to $0.24 \mathrm{eV}$, while the high-energy $e_{g}$ doublet appears above the Fermi level. At $\Gamma$ -

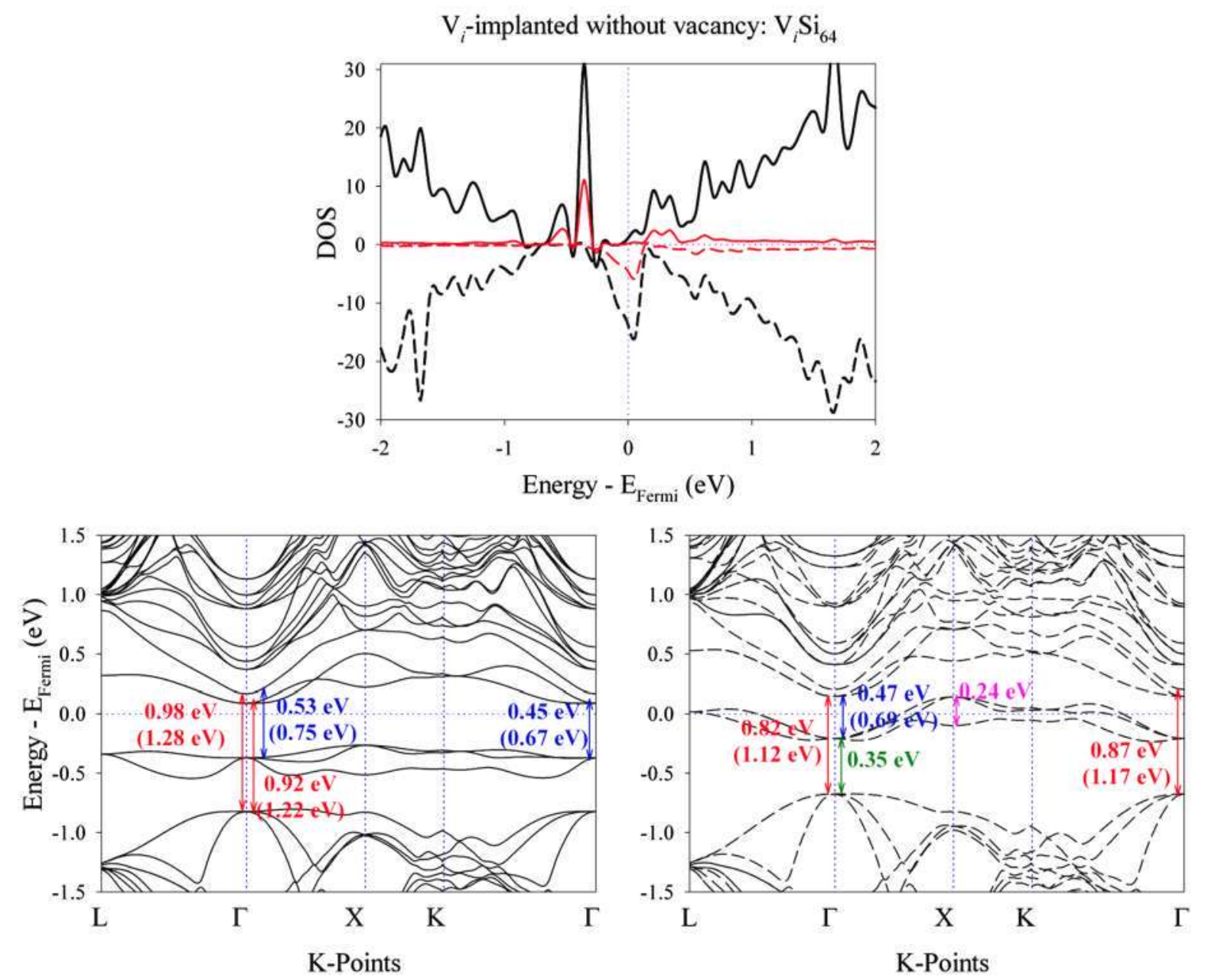

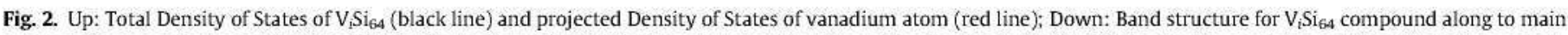

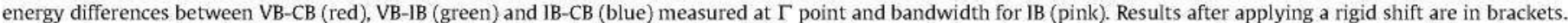

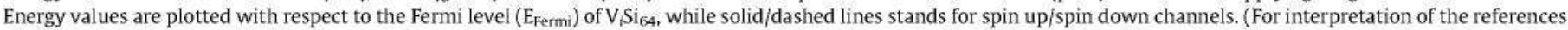
to colour in this figure legend, the reader is referred to the web version of this article.) 

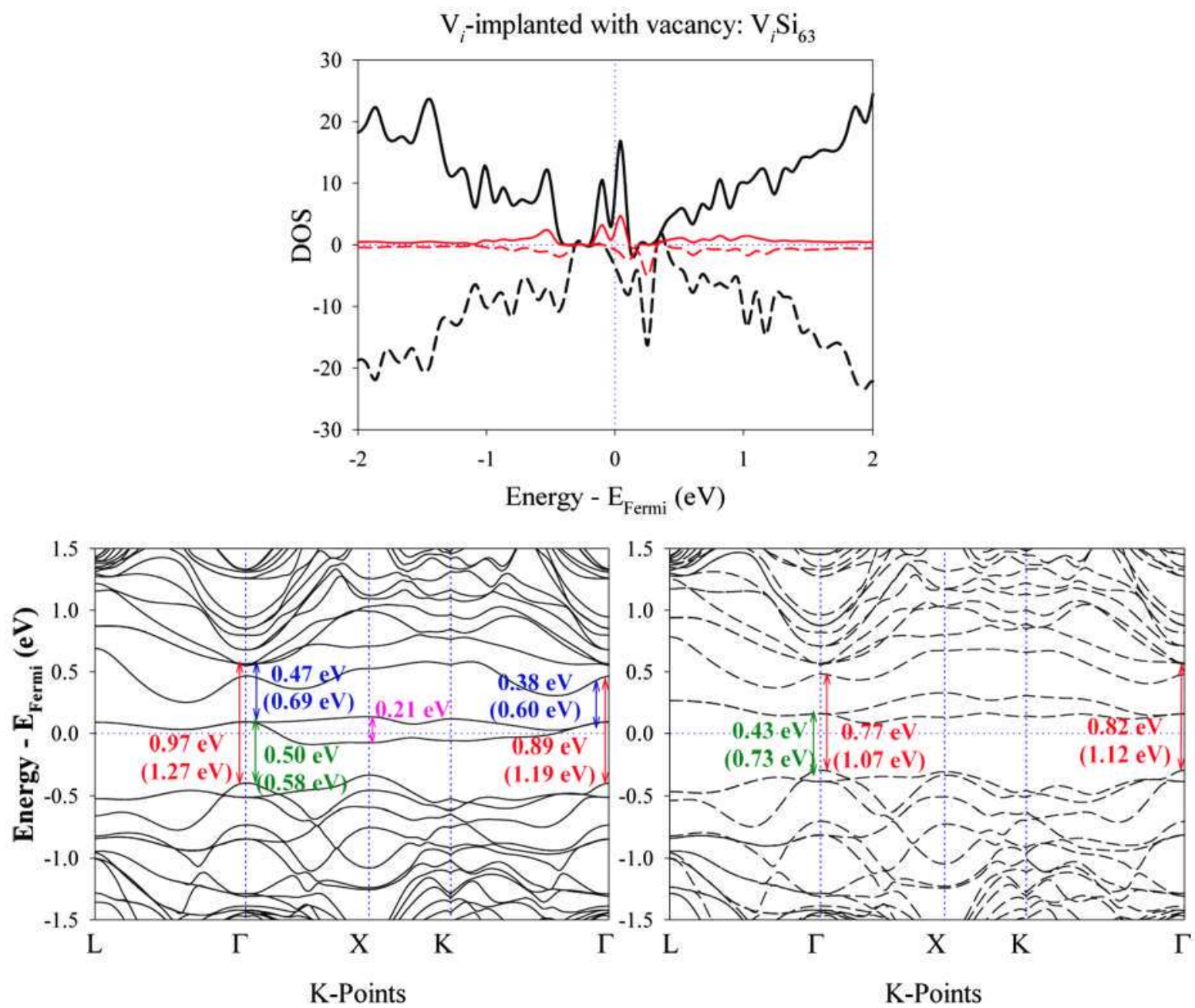

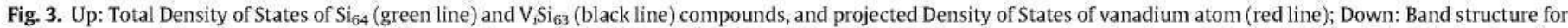

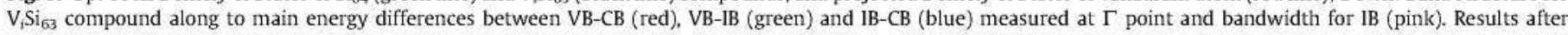

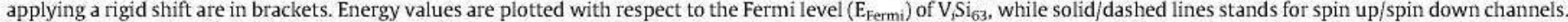
(For interpretation of the references to colour in this figure legend, the reader is referred to the web version of this article.)

point, the low energy doublet yields a difference of $0.35 \mathrm{eV}$, while the largest energy difference, $1.74 \mathrm{eV}(1.82 \mathrm{eV})$, is measured at Xpoint. Such transitions are labeled as CB-IB. The splitting energy between high energy $e_{g}$ doublet and low energy $t_{2 g}$ triplet (IB-CB energy difference) is equal to $0.47 \mathrm{eV}(0.69 \mathrm{eV})$. The $e_{g}$ doublet appears at $0.82 \mathrm{eV}(1.12 \mathrm{eV})$ over the $\mathrm{CB}$ of the host $\mathrm{Si}$. The energy difference between the $\mathrm{VB}$ and the $\mathrm{CB}$ is $0.87 \mathrm{eV}(1.17 \mathrm{eV})$, which is less important than that found for $\mathrm{V}_{S i} \mathrm{Si}_{63}$ compound. As concerns as spin up component, the triplet is located below the Fermi energy, while the doublet has larger energy than the Fermi level. The energy difference, measured at $\Gamma$-point, between the VB and $\mathrm{CB}$ is $0.98 \mathrm{eV}(1.28 \mathrm{eV})$, which is also lower than that found $\mathrm{V}_{\mathrm{Si}^{-}}$ implanted compound. In addition, filled $t_{2 g}$ levels could allow new transitions energies up to empty $e_{g}$ levels and $\mathrm{CB}$ of bulk-Si, with energies equal to $0.45 \mathrm{eV}(0.67 \mathrm{eV})$ and $0.53 \mathrm{eV}(0.75 \mathrm{eV})$, respectively. For clarity, these transitions are labeled as $\mathrm{IB}-\mathrm{CB}$, which easily allow to identity that they start from donor levels due to $\mathrm{V}$ atom (i.e., $t_{2 g}$ ). Finally, since $e_{g}$ doublet is very close to the $\mathrm{CB}$, new transitions (also labeled as $\mathrm{VB}-\mathrm{CB}$ ) with somewhat lower energy, $0.92 \mathrm{eV}(1.32 \mathrm{eV})$, would be also possible.

Among different $\mathrm{V}_{i} \mathrm{Si}_{63}$ structures defined based on the relative disposition between $\mathrm{V}$ atom and $\mathrm{Si}$ vacancy, only those one with the lowest formation energy $\left(v-c . V_{i} \mathrm{Si}_{63}\right)$ has been selected to assess the presence of both $\mathrm{V}$ atom and $\mathrm{Si}$ vacancies (Fig. 3). The vacancy implies that tetrahedral and octahedral coordination of $\mathrm{V}$ atom is distorted respect to the compound without vacancy, which leads to a more complex electronic structure. Thus, two sets of vanadium bands are located into the bandgap of the host. For spin up channel, there is a doublet located over the Fermi energy, which fulfills the requirements to play as $I B$, i.e., this is partially filled and isolated from both conduction and valence bands. This IB has a bandwidth $=0.21 \mathrm{eV}$. Main energy differences related with this $\mathrm{IB}$ are those labeled as VB-IB and IB-CB, with values equal to $0.50 \mathrm{eV}$ $(0.58 \mathrm{eV})$ and $0.47 \mathrm{eV}(0.69 \mathrm{eV})$, respectively, at $\Gamma$-point. There is also an empty band, located with an energy of $0.89 \mathrm{eV}(1.19 \mathrm{eV})$ over the VB and $0.38 \mathrm{eV}(0.60 \mathrm{eV})$ over the IB. Both levels forming the IB and this empty level would be related with the low energy $t_{2 \mathrm{~g}}$ triplet originated by the (distorted) octahedrical coordination of $V$ atom along to the vacancy, while high energy $e_{g}$ doublet is highly hybridized with unoccupied levels of bulk-Si. For spin down component, similar results are displayed in Fig. 3. Nonetheless, the $t_{2 g}$ triplet is empty, while the $e_{g}$ doublet appears again hybridized with the conduction bands coming from silicon. Hence, only energy differences taking into account $\mathrm{VB}$ as reference are displayed in Fig. 3, which lie between $0.43 \mathrm{eV}(0.73 \mathrm{eV})$ and $0.82 \mathrm{eV}$ $(1.12 \mathrm{eV})$. It should be noted that the bandgap for host $\mathrm{Si}$ is opened up to $0.97 \mathrm{eV}(1.27 \mathrm{eV}) / 0.82 \mathrm{eV}(1.12 \mathrm{eV})$ for spin up/down component, which is similar than to obtained in absence of Si vacancy. 


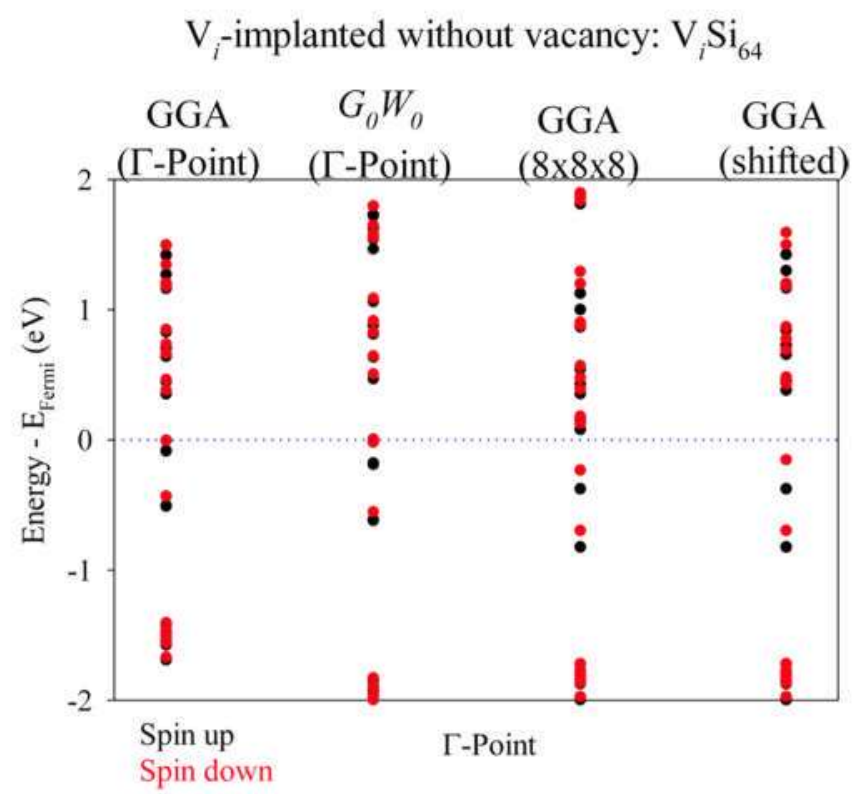

Fig. 4. Eigenvalues calculated for $\mathrm{V}_{i} \mathrm{Si}_{64}$ at $\Gamma$-point and those obtained after applying rigid shift.

\section{4. $G_{0} W_{0}$ results and bandgap correction}

Optical properties have been assessed through the imaginary part of the dielectric tensor as a sum over independent KohnSham transitions. Among other approaches, the "bandgap problem" related to DFT has been commonly bypassed by applying a scissor operator (a rigid shift) over the empty states to match the experimental bandgap of the host semiconductor. Though this practice could be adequate for bulk-Si, it neglects the effect that new (vanadium) bands can produce on the bandgap energy of the host semiconductor as well as the displacement to be applied to partially filled intermediate band. Concretely, we have calculated quasiparticle energies through $G_{0} W_{0}$ approach. Due to the high computational requirements, quasiparticle energies were only calculated for $\mathrm{Si}_{64}$ and $\mathrm{V}_{i} \mathrm{Si}_{64}$ at $\Gamma$-point. We are aware that these $G_{0} W_{0}$ results should be treated with caution. Nevertheless, in the case of $\mathrm{Si}_{64}$ (not shown), $G_{0} W_{0}$ yields a bandgap equal to $1.03 \mathrm{eV}$, which clearly improves DFT underestimation. Thus, results for $\mathrm{Si}_{64}$ will undergo a bandgap correction based on a rigid shift of $0.40 \mathrm{eV}$ applied over empty bands. Fig. 4 plots computed energies for $\mathrm{V}_{i} \mathrm{Si}_{64}$ at $\Gamma$-point by using GGA (GGA ( $\Gamma$-point)) and $G_{0} W_{0}$ $\left(G_{0} W_{0}(\Gamma\right.$-point $\left.)\right)$ approaches along to GGA energies obtained also at $\Gamma$-point with an $8 \times 8 \times 8$ sampling of the Brillouin zone (GGA $(8 \times 8 \times 8))$ and obtained energies after applying the rigid shift (GGA (shifted)). As concerns as $\mathrm{V}_{i}$-implanted compound, although the position of those bands in the vicinity of the Fermi level is affected by sampling of the Brillouin zone, similar energies are obtained for the key transitions, i.e., VB-CB, VB-IB and IB-CB. Based on $G_{0} W_{0}$ results, a rigid shift of $0.30 \mathrm{eV}$ should be applied to CB-VB transitions. The energy difference between VB and partially filled is also opened $(0.08 \mathrm{eV})$. Hence, a rigid shift of $0.08 \mathrm{eV}$ and $0.22 \mathrm{eV}$ will be applied to VB-IB and IB-CB transitions, respectively. These same rigid shifts will be also applied for the remaining $V$ implanted compounds.

\subsection{Optical properties}

Optoelectronic properties of V-implanted compounds have been studied through the imaginary component of the dielectric function (see Fig. 5). Based on $G_{0} W_{0}$ results, a rigid shift has been

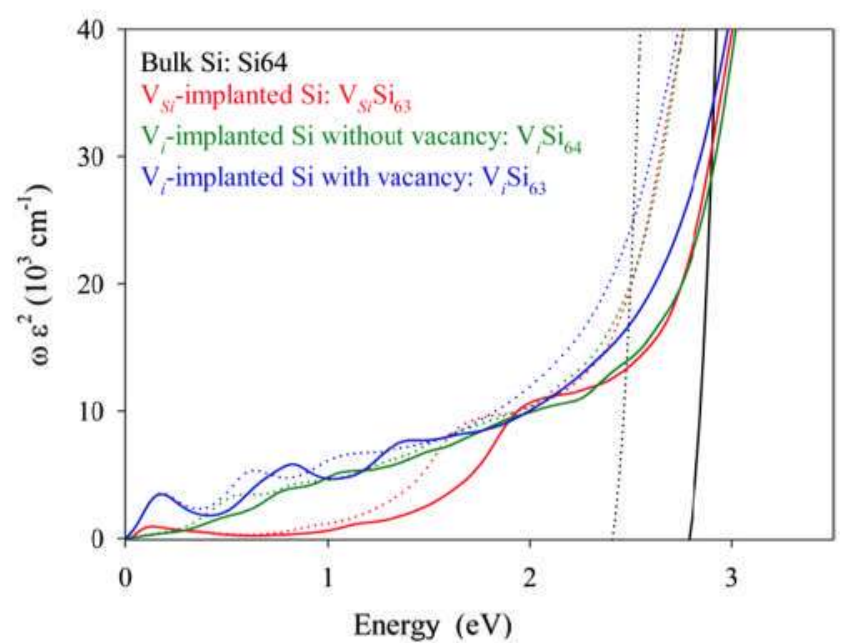

Fig. 5. Imaginary part of the dielectric functions calculated for bulk-Si and Vimplanted Si compounds. Results without applying the rigid shift are also displayed as dotted lines.

applied to correct the GGA underestimation of the bandgap. For $\mathrm{Si}_{64}$, our results agree with experimental absorption spectrum (except for those features related to indirect transitions and exciton effects) [53]. The absorption features of V-implanted compounds can be enhanced in the energy range below $2.8 \mathrm{eV}$. While for $\mathrm{V}_{S i}$-implanted compound there is an abrupt diminution of the absorption coefficient below $2.0 \mathrm{eV}$, both $\mathrm{V}_{i}$-implanted compounds display an improved absorption up to $0.20 \mathrm{eV}$. In addition, for $\mathrm{V}_{i^{-}}$ implanted compound with a $\mathrm{Si}$ vacancy $\left(\mathrm{V}_{i} \mathrm{Si}_{63}\right)$, three peaks located at roughly $0.20 \mathrm{eV}, 0.80 \mathrm{eV}$ and $1.20 \mathrm{eV}$ own larger intensity. The imaginary part of the dielectric function has been decomposed into the contributions from each type of transitions to verify the role of the $\mathrm{V}$ in the absorption enhancement (Figs. $4 \mathrm{~S}$ and 6). Note that from $2.8 \mathrm{eV}$, the main contribution comes from $\mathrm{VB}-\mathrm{CB}$ transitions of the host semiconductor. For all V-implanted compounds, those peaks located between $0.18 \mathrm{eV}$ and $0.25 \mathrm{eV}$ are due to IB-IB transitions. For, $\mathrm{V}_{S i}$-implanted $\mathrm{Si}$ (Fig. 4S, see supplementary information for a more detailed description), the broad peak centered at $2.0 \mathrm{eV}$ is mainly due to $\mathrm{CB}-\mathrm{VB}$ transitions.

For compounds with interstitial implantation ( $V_{i}$, Fig. 6), the absorption enhancement is extended up to $0.25 \mathrm{eV}$ and $0.18 \mathrm{eV}$ for $\mathrm{V}_{i} \mathrm{Si}_{64} \quad\left(\mathrm{~V}_{i}\right.$-implanted without vacancy) and $\mathrm{V}_{i} \mathrm{Si}_{63} \quad\left(\mathrm{~V}_{i^{-}}\right.$ implanted with a Si vacancy), respectively. These peaks are due to IB-IB transitions. For $\mathrm{V}_{\mathrm{i}}$-implanted compound in absence of silicon vacancies, the dielectric function gradually decreases between $2.8 \mathrm{eV}$ and $0.25 \mathrm{eV}$. VB-CB contributions gradually increase their intensity up to $2.8 \mathrm{eV}$, from where the largest contribution is due to VB-CB of the host. Note that VB-CB transitions start to appear at $1.10 \mathrm{eV}$, which is related to energy differences of $\approx 1.15 \mathrm{eV}$ shown in Fig. 2 for spin down component, measured between the $\mathrm{VB}$ and the $\mathrm{CB}$ as well as between the VB and empty $e_{g}$ states of $\mathrm{V}$ atom. Contributions from IB-CB transitions for spin up become visible at $\approx 0.65 \mathrm{eV}$ (in agreement with those energy differences labeled as IB-CB corresponding to energy differences between $t_{2 \mathrm{~g}}$ and $e_{g}$ states in Fig. 2 for spin up). This contribution yields two main peaks. The first one located at $0.75 \mathrm{eV}$ is assigned to IB-CB transitions due to the energy difference between filled $t_{2 g}$ states of $\mathrm{V}$ atom and $\mathrm{CB}$ of bulk-Si (see Fig. 2). The broad peak lying between $1.40 \mathrm{eV}$ and $2.50 \mathrm{eV}$ is due to electronic transition between occupied $t_{\mathrm{g} 2}$ donor levels of $\mathrm{V}$ atom and high energy empty bands. The peaks appearing at $\approx 0.70 \mathrm{eV}, 1.10 \mathrm{eV}$ and $1.80 \mathrm{eV}$ are assigned to IB-CB transitions for spin down. The former can be related to the energy difference of $0.69 \mathrm{eV}$ measured 

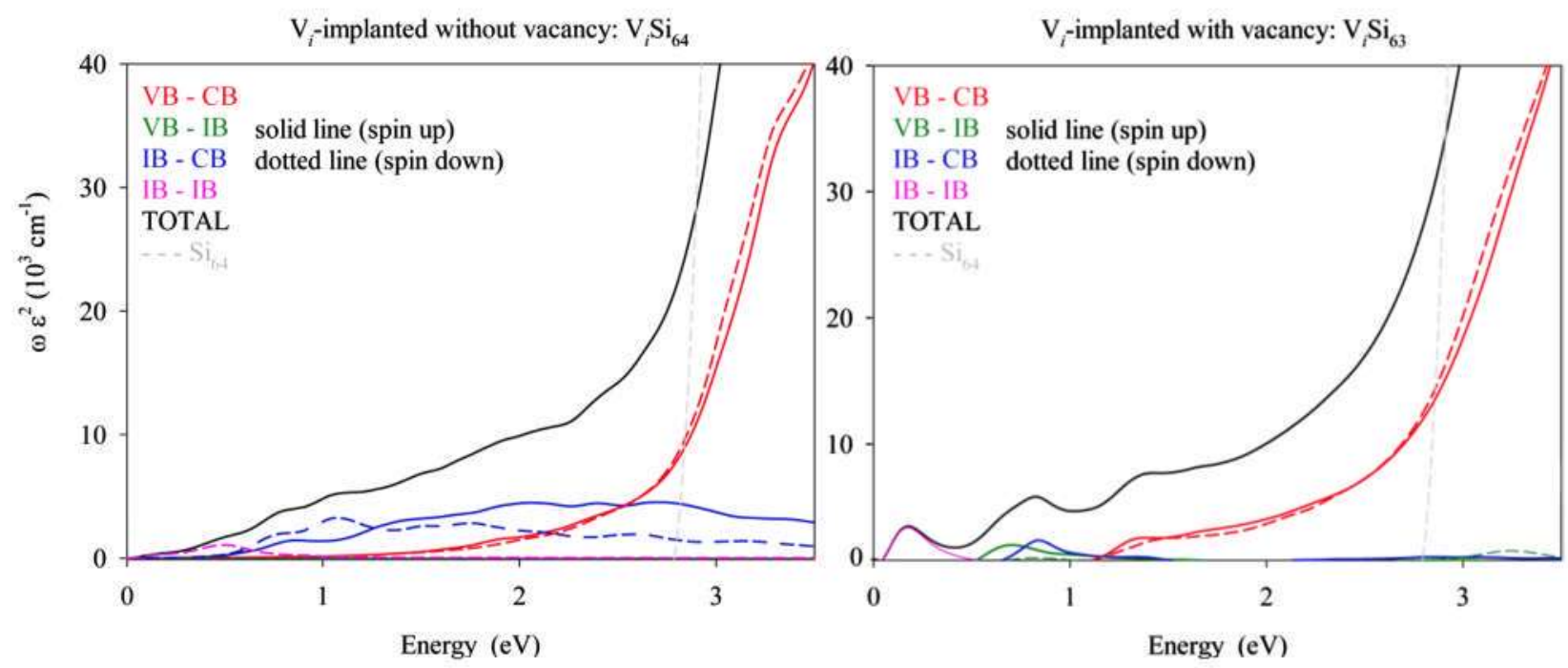

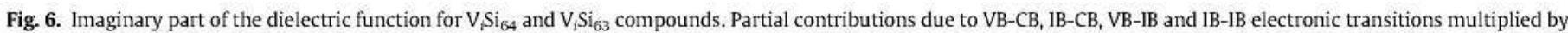
the frequency as well as the results for $\mathrm{Si}_{64}$ have been also included.

between the intermediate band (due to partially filled $t_{2 g}$ triplet) and high energy (empty) $e_{g}$ doublet in Fig. 2 for spin down, while the remaining two peaks are due to electronic transitions between partially filed $t_{2 \mathrm{~g}}$ levels of $\mathrm{V}$ atom and high energy empty bands.

In presence of both $\mathrm{V}_{i}$-implanted and $\mathrm{Si}$ vacancy $\left(\mathrm{V}_{i} \mathrm{Si}_{63}\right.$, see Fig. 6), the main enhancement of the absorption coefficient is due to two transitions involving contributions across the IB. As said, the main difference with the absorption features of $\mathrm{V}_{i^{-}}$ implanted compound in absence of a Si vacancy is the presence of differentiated peaks at $0.20 \mathrm{eV}, 0.80 \mathrm{eV}$ and $1.20 \mathrm{eV}$, wherein the first one is due to IB-IB transitions. It should be noted that $\mathrm{V}_{i^{-}}$ $\mathrm{Si}_{63}$ yields IB-IB transitions with the largest dielectric constant. The peak at $1.20 \mathrm{eV}$ is associated with vanadium-assisted VB-CB electronic transitions, which yields a very similar profile for both spin up and spin down components. VB-CB transitions emerge at $\approx 1.10 \mathrm{eV}$, in concordance with energy differences measured in Fig. 3 between VB and CB or empty states or V atoms. The peak at $0.80 \mathrm{eV}$ should be described as the sum of two contributions at roughly $0.84 \mathrm{eV}$ and $0.67 \mathrm{eV}$ due to IB-CB and VB-IB transitions, respectively. Hence, at high enough vanadium concentrations, infrared radiation would lead to free carriers able to enhance absorption sub-bandgap transitions across the IB located in the midgap of host semiconductor. As said in the introduction, hyperdoping with transition metals would also allow working at room temperature due to high thermal ionization of deep level impurities. Results here exposed for $\mathrm{V}_{i}$-implanted compounds are in agreement with results of García-Hemme et al., who found an important increase of the photoresponse with respect to a Si reference, which extends into the infrared regions, at room temperature [20]. Hence, experimental measured photoresponse in Vimplanted compounds can be explained based on new subbandgap transitions across the intermediate band coming from $\mathrm{V}$-implantation. Note that the formation of this intermediate band with the adequate properties is only possible for interstitial vanadium.

\section{Conclusions}

This works reports a detailed first principle study based on Density Functional Theory simulations on V-implanted Si compounds. For this purpose, crystal structures, formation energies $\left(E^{f}\right)$ and electronic properties were studied at substitutional $\left(\mathrm{V}_{S i}\right)$ and inter- stitial $\left(\mathrm{V}_{i}\right)$ vanadium implantations, as well as the effect of $\mathrm{Si}$ vacancies. The full relaxation of atomic structures yield high formation energy values for all implantation process, being $V_{i}$ in absence of Si vacancy the most favorable one. After considering that the main contribution from the energetic penalty to remove one $\mathrm{Si}$ atom, $E^{f}$ becomes lower than zero for interstitial implantation, i.e., interstitial implantation process turns out to be favorable. Regarding to electronic structures of V-implanted compounds, for $\mathrm{V}_{i}$-implanted compound in absence of Si vacancies $\left(\mathrm{V}_{i} \mathrm{Si}_{64}\right)$, the octahedral crystal field due the six second nearest $\mathrm{Si}$ atoms leads to splitting $3 d$ levels into low energy $t_{2 g}$ triplet and a high-energy $e_{\mathrm{g}}$ doublet, where the low energy $t_{2 \mathrm{~g}}$ triplet for spin down channel would fulfill the requirements to be defined as an intermediate band (IB). $\mathrm{V}_{i}$-implanted in presence of a Si vacancy also displays an IB due to $t_{2 g}$ levels for spin up channel. Nonetheless, the Si vacancy leads to only two $t_{2 \mathrm{~g}}$ level are occupied, while the other one is empty and highly in energy.

One of the main aims of this work is to shed some light on the absorption features of $\mathrm{V}$-implanted compounds and their potential as materials with infrared activity. In this work a scissor operator based on quasiparticle energies has been applied to overcome the underestimation of bandgap in DFT. Concretely, we performed the one-shot $G W$ approach $\left(G_{0} W_{0}\right)$. Although quasi particle energies where only computed at $\Gamma$-point, there is a clear improvement in the calculated bandgap for $\mathrm{Si}_{64}$. Previous works in literature have applied a rigid shift over the empty state to recover the experimental bandgap of the host semiconductor. Our $G_{0} W_{0}$ has revealed that the rigid shift applied to $\mathrm{V}$-implanted compounds could be different to the host material, as well as information on the correction for partially filled bands. After applying the rigid shift, our results point out that there is a considerable improvement in the absorption coefficient below $2.8 \mathrm{eV}$. In addition, $\mathrm{V}_{i}$-implanted compounds also exhibit adequate absorption features below $1.0 \mathrm{eV}$ due to different transitions across the intermediate band. Thus, our results offer a possible path to design new silicon-based materials with infrared absorption features.

\section{Acknowledgements}

This work was partially supported by the Comunidad de Madrid project MADRID-PV (S2013/MAE/2780) and by the Ministerio de Economía y Competitividad through the project BOOSTER (ENE2013-46624-C4-2-R). The authors acknowledge the computer 
resources and technical assistance provided by the Centro de Supercomputación and Visualización de Madrid (CeSViMa).

\section{Appendix A. Supplementary material}

Supplementary data associated with this article can be found, in the online version, at http://dx.doi.org/10.1016/j.commatsci.2017. 05.005 .

\section{References}

[1] X. Liu, B. Kuyken, G. Roelkens, R. Baets, R.M. Osgood, W.M.]. Green, Nat. Photon. $6(2012) 667$

[2] B.Y. Zhang, T. Liu, B. Meng, X. Li, G. Liang, X. Hu, Q.J. Wang, Nat. Commun. 4 (2013) 1811.

[3] A. Rogalski, Prog. Quantum Electron. 27 (2003) 59.

[4] S. Chakrabarti, A.D. Stiff-Roberts, X.H. Su, P. Bhattacharya, G. Ariyawansa, A.G. U. Perera, J. Phys. D Appl. Phys. 38 (2005) 2135.

[5] J.E. Carey, C.H. Crouch, M. Shen, E. Mazur, Opt. Lett. 30 (2005) 1773.

[6] S. Hu, P. Han, S. Wang, X. Mao, X. Li, L. Gao, Semicond. Sci. Technol. 27 (2012) 102002.

[7] M.T. Winkler, D. Recht, M.J. Sher, A.J. Said, E. Mazur, M.J. Aziz, Phys. Rev. Lett. $106(2011) 178701$.

[8] E. Ertekin, M.T. Winkler, D. Recht, A.J. Said, M.J. Aziz, T. Buonassisi, J.C Grossman, Phys. Rev. Lett. 108 (2012) 026401.

[9] A.J. Said, D. Recht, J.T. Sullivan, J.M. Warrender, T. Buonassisi, P.D. Persans, M.J. Aziz, Appl. Phys. Lett. 99 (2011) 073503.

[10] M. TabbaI, T. Kim, D.N. Woolf, B. Shin, M.J. Aziz, Appl. Phys. A 98 (2009) 589.

[11] M.A. Sheehy, B.R. Tull, C.M. Friend, E. Mazur, Mater. Sci. Eng., B 137 (2007) 289.

[12] J.T. Sullivan, C.B. Simmons, J.]. Krich, A.J. Akey, D. Recht, M.J. Aziz, J. Appl. Phys. $114(2013) 103701$

[13] C.B. Simmons, A.J. Akey, J.P. Mailod, D. Recht, M.J. Aziz, Adv. Funct. Mater. 24 (2014) 2852.

[14] T. Sadoh, H. Nakashima, T. Tsurushima, J. Appl. Phys. 72 (1992) 520.

[15] J. Olea, A. del Prado, D. Pastor, L. Mártil, G. González-Díaz, I. Appl. Phys, 109 (2011) 113541

[16] E. García-Hemme, R. García-Hernansanz, J. Olea, D. Pastor, A. del Prado, L. Mártil, G. González-Díaz, Appl. Phys. lett. 104 (2014) 211105.

[17] E. Antolín, A. Martí, J. Olea, D. Pastor, G. González-Díaz, I. Mártil, A. Luque, Appl. Phys. Lett. 94 (2009) 042115.

[18] E. Pérez, H. Castán, H. García, S. Dueñas, L. Bailón, D. Montero, R. GarcíaHernansanz, E. García-Hemme, J. Olea, G. González-Díaz, Appl. Phys, Lett. 106 (2015) 022105

[19] K. Sánchez, I. Aguilera, P. Palacios, P. Wahnón, Phys. Rev. B 79 (2009) 165203.
[20] E. García-Hemme, R. García-Hernansanz, ]. Olea, D. Pastor, A. del Prado, ]. Mártil, G. González-Díaz, Appl. Phys. Lett. 103 (2013) 032101.

[21] R. Chen, B. Fan, M. Pan, Q. Cheng, C. Chen, Mater, Lett. 163 (2016) 90.

[22] J.P. Mailoa, A.J. Akey, C.B. Simmons, D. Hutchinson, J. Mathews, J.T. Sullivan, D. Recht, M.T. Winkler, J.S. Williams, J.M. Warrender, P.D. Persans, M.J. Aziz, Nat. Commun. 5 (2014) 3011.

[23] S.H. Oh, K.V. Benthem, S.I. Molina, A.Y. Borisevich, W. Luo, P. Werner, N. Zakharov, D. Kumar, S.T. Pantelides, S.J. Pennycook, Nano lett. 8 (2008) 1016.

[24] N. López, L.A. Reichertz, K.M. Yu, K. Campman, W. Walukiewicz, Phys, Rev, Lett. 106 (2011) 028701.

[25] A. Luque, A. Marti, C. Stanley, Nat. Photon. 6 (2012) 146.

[26] X. Dong, X. Song, Y. Wang, J. Wang, Appl. Phys. Express 8 (2015) 081302.

[27] P.H. Huang, C.M. Lu, Sci. World J. 2014 (2014) 8.

[28] S. Lany, A. Zunger, Phys. Rev. B 78 (2008) 235104

[29] M.K.Y. Chan, G. Ceder, Phys. Rev. Lett. 105 (2010) 196403.

[30] I. Hedin, Phys. Rev. 139 (1965) A796.

[31] J.P. Perdew, K.B. Ernzerhof, Phys. Rev. Lett. 77 (1996) 3865.

[32] G. Kresse, J. Hafner, Phys. Rev. B 47 (1993) 558.

[33] G. Kresse, J. Furthmüller, Phys. Rev. B 54 (1996) 11169.

[34] P.E. Blöchl, Phys. Rev. B 50 (1994) 17953.

[35] G. Kresse, D. Joubert, Phys. Rev. B 59 (1999) 1758

[36] H.J. Monkhorst, J.D. Pack, Phys. Rev. B 13 (1976) 5188.

[37] M. Methfessel, A.T. Paxton. Phys. Rev. B 1989 (40) (1989) 3616.

[38] P.E. Blöchl, O. Jepsen, O.K. Andersen, Phys. Rev. B 49 (1994) 16223.

[39] K. Sánchez, I. Aguilera, P. Palacios, P. Wahnón, Phys. Rev. B 82 (2010) 165201.

[40] Furthmüller F. Calculate the (optical) dielectric function and joint density of states. Available at: http://www.freeware.vasp.de/VASP/optics/optics.F.

[41] R. Lucena, 1. Aguilera, P. Palacios, P. Wahnón, J.C. Conesa, Chem. Maters. 20 (2008) 5125.

[42] W.G. Aulbur, L. Jönsson, J.W. Wilkins, Quasiparticle calculations in solids, by H. Ehrenreich and F. Saepen, Solid State Physics, Academic Press, 1999.

[43] C.R. Hubbard, H.E. Swanson, F.A. Mauer, J. Appl. Cryst. 8 (1975) 45.

[44] C.G. Van de Walle, J. Neugebauer, ]. Appl. Phys. 95 (2004) 3851.

[45] A. Luque, A. Marti, E. Antolín, C. Tablero, Physica B 382 (2006) 320.

[46] D. Pastor, J. Olea, A. Muñoz-Martín, A. Climent-Font, 1. Mártil, G. GonzálezDíaz, J. Appl. Phys. 112 (2012) 113514

[47] P. Palacios, P. Wahnón, S. Pizzinato, J.C. Conesa, J. Chem. Phys. 124 (2006) 014711.

[48] P. Palacios, K. Sánchez, J.C. Consesa, P. Wahnón, Phys. Status Solidi A 203 (2006) 1395.

[49] P. Palacios, K. Sánchez, J.C. Conesa, J.J. Fernández, P. Wahnón, Thin Solid Films 515 (2007) 6280

[50] Y. Seminóvski, P. Palacios, J.C. Conesa, P. Wahnón, Comput. Theor. Chem. 975 (2011) 134.

[51] Y. Hinuma, A. Grüneis; G. Kresse, F. Ob, Phys. Rev. B 90 (2014) 155405.

[52] X. Liu, L. Li, Q. Li, Y. Li, F. Lu, Mater. Sci. Semicond. Process. 16 (2013) 1369.

[53] M.A. Green, Sol. Energy Mater. Sol. Cells 92 (2008) 1305. 